\title{
On the Insolation of a Sun of Sensible Magnitude.
}

\author{
By A. Ritchie Scott, B.Sc.
}

\begin{abstract}
[ABstract.]
Many theories have been advanced to account for a presumed uniformity in the temperature of the earth in past ages, and one of the most recent is that advanced by Sir John Murray in the Summary Volumes of the Challenger Report. A careful study of the distribution of marine fauna showed the existence of remarkably similar organic forms in the Arctic and Antaretic regions which were entirely unknown in intermediate waters. The existence, also, of ancient corals in Polar seas points to a high Polar temperature at some remote period of the earth's history. These facts combined lead us to consider it probable that in some past age the earth's air temperature was high and uniform. Sir John Murray, founding upon a paper by $\mathbf{M}$. Blandet, suggests that this may have been due to the fact that life may have been possible on the earth long before the sun, in its cosmic development, had shrunk to its present dimensions. At present, at the solstice the sun only shines up to the Polar Circle at one of the poles, but the paleocosmic sun may have been so large that at the solstice the limb of the sun might still have shone on the pole and thereby kept up the Polar temperature. The present paper is an attempt to work out, as far as possible, the redistribution of temperatures under such circumstances.

For simplicity, we will assume that the large sun is of such a size that at the solstice its upper limb just illumines the poles--i.e., its angular semi-diameter will be $23^{\circ} 27^{\prime}$. We may also assume that it emits the same total energy as at present.

While the whole sun is above the horizon the rate of insolation will obviously be independent of the size of the solar disc. When, however, the sun is setting, the upper limb of the sun will continue to insolate any given point on the earth's surface for a considerable time after the centre of the solar disc has disappeared, and the same phenomenon will occur in opposite order during sunrise. During such intervals the given point on the earth's surface will receive a quantity of energy beyond what it gets at present from a (practically) point sun. Putting this otherwise, the large sun will
\end{abstract}


shine round the hemisphere further than at present $\left(23 \frac{1}{2}^{\circ}\right.$ on our present assumption). In order to calculate the rate of insolation from the sun at any given altitude, the solar disc was divided into elementary strips parallel to the horizon; the insolation from each strip is proportional to the length of the strip multiplied by the cosine of the angle of inclination. The insolation from the whole sun was then got by integrating from the horizon to the upper limb of the sun. The integral being irreducible, the integration was graphical.

A formula was next investigated, giving the relation between the height of the sun and the time of day. The hours after noon were then laid down as abscissa and the rate of insolation at the calculated height as ordinates, and a curve drawn. The area of the curve by planimeter gave the energy incident from noon to sunset.

In order to get comparable results, the insolation due to a point sun was plotted simultaneously with that of the sun of sensible magnitude, and measured under the same conditions.

\begin{tabular}{|c|c|c|c|c|c|c|}
\hline \multirow{2}{*}{ LAT. } & \multicolumn{2}{|c|}{ SUMMER SOLSTICE. } & \multicolumn{2}{|c|}{ EQUINOX. } & \multicolumn{2}{|c|}{ WINTER SOLSTICE. } \\
\hline & Laroe BUn. & PoINT SUN. & Large 8Un. & PonNT SUN. & Laras Sun. & Point Son. \\
\hline $0^{\circ}$ & 950 & 920 & 1027 & 1000 & 950 & 920 \\
\hline $10^{\circ}$ & 1038 & 1013 & 1009 & 984 & 818 & 793 \\
\hline $20^{\circ}$ & 1113 & 1087 & 965 & 940 & 685 & 661 \\
\hline $23^{\circ} 27^{\prime}$ & 1126 & 1098 & 934 & 908 & 629 & 602 \\
\hline $30^{\circ}$ & 1160 & 1130 & 892 & 864 & 535 & 503 \\
\hline $40^{\circ}$ & 1183 & 1146 & 798 & 766 & 385 & 350 \\
\hline $45^{\circ}$ & 1205 & 1158 & 725 & 694 & 311 & 264 \\
\hline $50^{\circ}$ & 1203 & 1160 & 686 & 649 & 248 & 190 \\
\hline $60^{\circ}$ & 1203 & 1129 & 521 & 471 & 127 & 75 \\
\hline $66^{\circ} 33^{\prime}$ & 1213 & 1146 & 474 & 406 & 73 & 0 \\
\hline $70^{\circ}$ & 1215 & 1169 & 421 & 344 & 55 & 0 \\
\hline $80^{\circ}$ & 1242 & 1234 & 324 & 163 & 20 & 0 \\
\hline $90^{\circ}$ & 1267 & 1267 & 283 & 0 & 0 & 0 \\
\hline
\end{tabular}

This table sums up the results of the work. A complete discussion would involve the summing of the daily insolation at each latitude 
throughout the whole year, an amount of work which did not seem warranted by the importance of the calculation, particularly as the brosd result is sufficiently indicated by these numbers.

The step from incident energy to air temperature is difficult, if not impossible, in the present state of meteorology ; nevertheless, the comparatively small increase in the amount incident radiant energy, on account of the size of the sun, does not seem to warrant our accepting a sun of sensible magnitude as a sole cause for uniformity of temperature.

Some other interesting figures may be readily got from our data. Since the sun's polar distance and zenith distance are equal at the pole, the zenith distance may be approximately expressed as a simple function of the time of year. Plotting the time (in days from equinox) as abscissa and the insolation at each date as ordinates, we find graphically that the energy incident at the pole during one whole year under the large sun would be to that at present as $1173: 1000$.

Again, consider any point on the earth's surface with the sun in the zenith; here the rate of insolation is a maximum. Around this point we may draw concentric small circles at all points, of any one of which the rate of insolation is the same. Imagine now the energy incident throughout any short time to be piled up on the earth's surface; we will now have a solid of revolution whose volume will be proportional to the rate at which the earth is intercepting radiant energy. The ratio of the rates at which energy is intercepted from the large sun and the small sun respectively is $1072: 1000$. 